\title{
Calidad del registro del certificado de defunción en un hospital pediátrico de Lima, Perú
}

\section{Quality registration of the death certificate in a hospital pediatric of Lima, Perú}

\author{
Nieves Zeta-Ruiz ${ }^{1}$, Carlos Soncco ${ }^{2}$ \\ ${ }^{1}$ Alumna de postgrado, Universidad Nacional Mayor de San Marcos, Lima, Perú. \\ ${ }^{2}$ Médico especialista en Gestión en Salud. Hospital de Emergencias Pediátricas, Lima, Perú.
}

\section{Correspondencia}

Nieves del Milagro Zeta Ruiz

Teléfs. 68221207 - 968961670

Direcc. Conjunto Habitacional

Residencial FAP La Cruceta III etapa

Block 21 Dpto No 402 Santiago de

Surco.

nieves.zeta@unmsm.edu.pe

Recibido: 4 de abril 2017

Aceptado: 27 de agosto 2017

Conflictos de interés: Los autores declaran no tener ningún conflicto de interés

Financiamiento: Este trabajo de investigación ha sido autofinanciado

Citar como: Zeta-Ruiz N, Soncco C. Calidad del registro del certificado de defunción en un hospital pediátrico de Lima, Perú. An Fac med. 2017;78(4):405-8 DOI: http://dx.doi.org/10.15381/ anales.v78i4.14261

\section{An Fac med. 2017;78(4):405-8 / http://dx.doi.org/10.15381/anales.v78i4.14261}

\section{Resumen}

Introducción. Los certificados de defunción son documentos legales que cuentan con datos epidemiológicos y estadísticos necesarios para la toma de decisiones a nivel hospitalario, siendo de vital importancia el correcto llenado de este documento. Objetivo. Determinar la calidad del registro del Certificado de Defunción en el Hospital de Emergencias Pediátricas durante el periodo 2010-2014. Diseño. Estudio descriptivo y transversal. Lugar. Hospital de Emergencias Pediátricas, Lima - Perú. Materiales. Se incluyeron 60 certificados de defunción emitidos durante los años 2010 a 2014. Intervenciones. Se utilizó una ficha siguiendo los criterios utilizados en un estudio peruano anterior. Principales medidas de resultados. Se evaluó la calidad de los certificados empleando una escala ordinal de cuatro categorías; buena, regular, mala y pésima calidad. Resultados. De los 60 certificados de defunción evaluados, más del $50 \%$ fue de mala y pésima calidad, alcanzando solo el $47 \%$ de regular calidad. Conclusiones. El mayor porcentaje de certificados de defunción presentan mala y pésima calidad, siendo necesario capacitar a los profesionales médicos en el correcto llenado de este documento. Además sería importante implementar el uso del certificado de defunción electrónico, a fin que los decisores en salud puedan contar oportunamente con los correspondientes datos epidemiológicos y estadísticos.

Palabras Clave. Certificado de Defunción; Calidad.

\section{Abstract}

Introduction. Death Certificates are legal documents which have epidemiological and statistics data needed for decision making at the hospital level, being of vital importance the correct filling of this document. Objective. To determine the quality of the registration of the Certificate of Death in the Emergencias Pediatricas Hospital during the period 2010-2014. Design. descriptive and cross-sectional study. Place. Emergencias Pediátricas Hospital, Lima - Perú. Materials. 60 death certificates were included during the years 2010 to 2014. Interventions. A file chart was used following the criteria used in a previous Peruvian study. Main outcome measures. The quality of the certificates was evaluated using an ordinal scale of four categories: Good regular, bad and worst quality. Results. Of the 60 death certificates evaluated, more than $50 \%$ were of bad and worst quality, reaching only $47 \%$ of regular quality. Conclusions. The highest percentage of death certificates present bad and worst quality, being necessary to train the medical professionals in the correct filling of this document. Besides, it would also be important to implement the use of the electronic death certificate, so that health decision-makers can count on timely epidemiological and statistical data.

Keywords. Death certificate; Quality. 


\section{INTRODUCCIÓN}

En casi todos los países una de las obligaciones del médico es emitir el Certificado de Defunción, autorizado por las leyes o normas de cada país para certificar la muerte dada sus competencias ${ }^{(1)}$.

Los Certificados de Defunción son de valor legal, epidemiológico y estadísti$\mathrm{co}$, teniendo doble finalidad: Registrar oficialmente la muerte, y ser la fuente primaria de las estadísticas de mortalidad. Así pues, los datos estadísticos y epidemiológicos generados y obtenidos mediante el Certificado de Defunción son de gran utilidad para conocer la situación de salud poblacional ${ }^{(2,3)}$. En ese sentido es importante que el médico sea competente en el correcto registro de las causas de muerte, que garantizará mejor que cualquier otro factor la calidad de las estadísticas de mortalidad, evitando que se tomen decisiones erróneas o insuficientes en todos los campos de la actividad del Estado y de la economía en general, pero primordialmente en el de la Salud, pues ningún otro sector utiliza los datos de mortalidad y de causas de muerte con la extensión y con la frecuencia con que lo hace el sector de la Salud ${ }^{(1)}$.

Es importante que los médicos comprendan que la certificación de una defunción no es sólo un trámite, es una oportunidad para dejar testimonio de la muerte de una persona en cuanto a las causas que la provocaron y a los factores sociodemográficos y de salud asociados a ella ${ }^{(1)}$, que servirán para analizar la situación de salud y realizar intervenciones a partir de ello.

A pesar de la importancia de la correcta certificación de defunción, diversos estudios nacionales e internacionales muestran una elevada proporción de certificados de defunción incompletos o con errores ${ }^{(4-9)}$ que repercuten en la mala calidad de los datos registrados y que la información obtenida sea de poca utilidad en la toma de decisiones a nivel local, regional y nacional. Es posible que uno de los factores que ocasionan esta deficiencia sea que los médicos no reciben las competencias necesarias para el correcto llenado del Certificado de Defunción durante su formación profe- sional, con frecuencia elaboran malos certificados, al ser enseñados por otros médicos que también desconocen el correcto llenado, sin supervisión ni revisión periódica por parte de algún profesional con conocimiento de la materia, con lo cual se perpetúa la errónea certificación de las muertes ${ }^{(1)}$.

La importancia y trascendencia de realizar una correcta certificación de la muerte ha sido señalada desde hace mucho tiempo por varios autores (10). En tal sentido, en nuestro país en el año 2009, el Ministerio de Salud (MINSA), a través del Oficina General de Estadística e Informática del Ministerio de Salud, implementó el "Manual para el Ilenado del formulario de defunción" con la finalidad de contribuir a mejorar el llenado de los datos del formulario de defunción para la producción de las estadísticas vitales, oportunas, completas, y de calidad (11). Sin embargo, dos estudios nacionales realizados en las ciudades de Ica y Chiclayo han demostrados errores en el llenado de certificados de defunción ${ }^{(8,9)}$. En la capital peruana, donde pudiese estimarse que el personal médico está capacitado en el correcto llenado de los certificados de defunción, no se han realizado estudios similares, considerándose necesario realizar esta investigación, a fin de contribuir a mejorar la calidad y la confiabilidad de las estadísticas de mortalidad en nuestro país, al ser uno de los pilares fundamentales en la toma de decisiones en salud pública.

El objetivo fue determinar la calidad del registro del Certificado de Defunción en el Hospital de Emergencias Pediátricas de Lima, Perú. Adicionalmente, identificar los errores más frecuentes en el llenado del certificado de defunción y determinar la frecuencia de los errores de forma y concepto en el llenado del Certificado de Defunción en el mencionado hospital.

\section{MÉTODOS}

Estudio de un diseño no experimental tipo transversal. La población de estudio estuvo conformada por las historias clínicas de los pacientes que fallecieron en el Hospital de Emergencias Pediátricas durante el periodo 2010- 2014, se consideraron criterios de exclusión: Historias clínicas de los pacientes fallecidos que no fueron ubicadas, certificados sin la parte superior desglosable y los casos de pacientes que pasaron a la morgue para ser sometidos a necropsia. Finalmente, se obtuvo una muestra conformada por 60 historias clínicas con sus respectivos certificados de defunción.

La técnica que se empleó en la investigación es el análisis documental, en base al certificado de defunción y la historia clínica. Para evaluar la calidad del registro del certificado de defunción se utilizó una ficha siguiendo los criterios utilizados en un estudio peruano anterior ${ }^{(8)}$.

Para revisar los certificados de defunción y las historias clínicas de los pacientes fallecidos se solicitó permiso a la Dirección del Hospital de Emergencias Pediátricas, quien aprobó la realización del estudio.

\section{RESULTADOS}

La Oficina de Estadística del Hospital de Emergencias Pediátricas proporcionó una data de 146 pacientes fallecidos en el mencionado hospital, de quienes se entregaron historias clínicas de 120 pacientes, no ubicándose 26. De las 120 historias entregadas se excluyeron 60: 27 porque pasaron a la morgue para realización de necropsia; 27 estaban incompletas al no contar con la parte superior desglosable; 4 porque no contaban con el certificado de defunción; 1 al tratarse de un paciente fallecido en otro hospital y 1 al ser un paciente vivo que ingresó para un procedimiento médico. En total la muestra estuvo conformada por 60 Certificados de Defunción.

Del total de Certificados de Defunción analizados, ninguno fue de buena calidad. El mayor porcentaje de certificados fue de regular y pésima calidad (47\%, respectivamente). Mientras que el $7 \%$ fue de mala calidad.

En el $100 \%$ de los certificados de defunción no todos los rubros estaban correctamente llenados. En el 75\% de los Certificados de Defunción se consignaban las causas básica, intermedia y final 
Tabla 1. Evaluación de calidad del certificado de defunción.

\begin{tabular}{lc}
\hline Criterios de evaluación & $\%$ \\
\hline Forma & \\
\hline Cuenta con todos los rubros correctamente llenados & $0 \%$ \\
Uso de abreviaturas & $7 \%$ \\
\hline Concepto & $75 \%$ \\
\hline $\begin{array}{l}\text { Diagnósticos de causa básica, intermedia y final están consignados } \\
\text { Diagnósticos de causa básica, intermedia y final consignados en secuencia lógica. }\end{array}$ & $68 \%$ \\
Presenta como único diagnóstico paro cardiorrespiratorio o paro cardiaco. & $0 \%$ \\
\hline Causa básica registrada en desglosable que va a Registro Civil & $45 \%$ \\
\hline $\begin{array}{l}\text { Diagnóstico de causa básica correcto en el desglosable que va a registro civil. } \\
\text { Diagnóstico de causa básica coincidente tanto en el desglosable que va a registro civil } \\
\text { como a estadística MINSA. }\end{array}$ & $38 \%$ \\
\hline
\end{tabular}

Fuente: Certificados de defunción emitidos en Hospital de Emergencias Pediátricas durante el 2010-2014.

de defunción y en el $68 \%$ de los certificados analizados las causas estaban consignadas en secuencia lógica. El diagnóstico de causa básica fue correcto en el desglosable que va a registro civil, en el $45 \%$ de los certificados.

Del total de certificados evaluados, las omisiones más frecuentes son no registrar los CIE 10 de los diagnósticos consignados como causas de defunción (82\%) y los intervalos aproximados entre el comienzo de la enfermedad y la muerte (95\%).

\section{DISCUSIÓN}

Los resultados encontrados en el presente estudio son preocupantes ya que ninguno de los certificados de defunción fue de buena calidad y esto se debió a que no cumplieron criterios de forma, ya que no tenían todos los rubros correctamente llenados, siendo las omisiones más frecuentes el no registro de CIE 10 y del intervalo entre el comienzo de la enfermedad y la muerte. Es necesario precisar que de haberse cumplido con el correcto llenado de todos los rubros, los certificados de regular calidad (47\%) hubiesen sido clasificados como de buena calidad. En la evaluación de criterios de concepto, si bien los diagnósticos de causa básica, intermedia y final estuvieron consignados en el $75 \%$ de los certificados, solo el $68 \%$ lo estuvo en secuencia lógica, lo que evidencia la necesidad de capacitación a los profesionales médicos. Respecto del registro de "paro cardiorrespiratorio" o "paro cardiaco" como causa de defunción, según la Organización Panamericana de la Salud, cuando se anota ese modo de morir sin que figure ninguna otra causa, es considerado como una causa mal definida ${ }^{1}$; sin embargo, en el presente estudio ningún certificado consignó como único diagnóstico "paro cardiorrespiratorio" o "paro cardiaco". Un resultado preocupante es que la causa básica consignada en el desglosable que va a registro civil y la consignada en el desglosable utilizado para información estadística del MINSA coincide solo en el $38 \%$ de los certificados, pudiendo indicar que no se conocen realmente las implicancias de este documento.

En el presente estudio la totalidad de certificados de defunción no contó con todos los rubros correctamente llenados, valor que difiere de un estudio realizado en México ${ }^{(2)}$ en que Hernández evidenció que el porcentaje de certificados con espacios sin llenar fue de $26,1 \%$. Asimismo el uso de abreviaturas en el llenado del certificado fue de $7 \%$ en el presente estudio, valor menor al encontrado por Hernández (17\%). Al comparar los resultados del presente estudio con otro estudio nacional, se evidencia que al igual que el estudio de Soto-Cáceres ${ }^{(8)}$, los certificados de pésima calidad representan el mayor porcentaje, aunque en el presente estudio el porcentaje es mayor $(47 \%$, mientras que en el estudio citado representa el 34,5\%). Asimismo en el mencionado estudio el porcentaje de certificados de buena calidad fue de $33,8 \%$, a diferente de este estudio en que ninguno de los certificados tuvo dicha calificación. Lo que puede deberse a que en el Hospital Nacional Nacional Almanzor Aguinaga Asenjo del departamento de Chiclayo se han realizado dos estudios previos y diversas intervenciones educativas, a fin de mejorar el registro de Certificado de Defunción. El porcentaje de certificados en que los diagnósticos estaban identificados y consignados en secuencia lógica fue de $68 \%$, mayor al encontrado en dos estudios nacionales: $56 \%$ hallado por Soto-Cáceres en Chiclayo ${ }^{(8)}$ y 32,1\%, por Valdez en Ica ${ }^{(9)}$. En el $45 \%$ de los certificados se consignó correctamente la causa básica en el desglosable del registro civil, porcentaje menor al hallado por Soto-Cáceres (71\%). El registro del correcto diagnóstico en el desglosable es importante, ya que uno de los fines del Certificado de Defunción es el legal, para inscribir la defunción en el Registro Civil y se autorice la inhumación, la cremación u otro procedimiento de destino final de los restos ${ }^{(1)}$. En el 38\% de los certificados no coincidió la causa básica consignada en el desglosable que va a Registro Civil (RENIEC) con la que se registró para estadística del MINSA, porcentaje similar al encontrado por Soto-Cáceres ${ }^{(8)}$, lo que indica la necesidad de concientización a los profesionales médicos sobre la utilidad de este documento con implicancias legales, epidemiológicas y estadísticas.

Una de las limitaciones en la realización del presente estudio fue que no se ubicaron el $17,8 \%$ de las historias clínicas solicitadas, asimismo el $18,5 \%$ de las historias clínicas solicitadas contenían solo la parte inferior de los certificados de defunción, no pudiéndose evaluar la coincidencia de la causa básica registrada en los desglosables superior e inferior del Certificado de Defunción, que son utilizados por RENIEC y el MINSA.

En conclusión, los Certificados de Defunción no están siendo llenados correctamente por los profesionales médicos del Hospital de Emergencias Pediátricas, evidenciándose errores no solo de concepto sino también de forma, asimismo la información contenida en la parte superior de este documento que será enviada a RENIEC y la utilizada para es- 
tadística del MINSA no coincide en más de la tercera parte de los Certificados de Defunción evaluados.

Se recomienda capacitar a los profesionales médicos del Hospital de Emergencias Pediátricas para que puedan registrar correctamente los datos solicitados en el certificado de defunción, por las implicancias de este documento. Asimismo, sería necesario implementar el certificado de defunción electrónico, que permita que el acceso oportuno de la información para la toma de decisiones.

\section{AGRADECIMIENTOS}

Al Hospital de Emergencias Pediátricas por su valioso apoyo y brindar las facilidades para la realización del presente estudio.

\section{REFERENCIAS BIBLIOGRÁFICAS}

1. Organización Panamericana de la Salud. Curso virtual sobre el correcto llenado del Certificado de Defunción. [Internet]. 2014 [citado 16 de marzo de 2016]. Recuperado a partir de: http:// es.slideshare.net/pomicin/certificado-de-defuncincorrecto-llenado.

2. Hernández C. Calidad del Ilenado del Certificado de Defunción y muerte fetal en un hospital de segundo nivel en México. Gac. int. cienc. Forense. 2014; 11:18-24.

3. Ministerio Público. Directiva Nº05-99-MP-FNIML/GT "Normas para el Uso del Certificado de Defunción en el Instituto de Medicina Legal" [Internet]. 1999 [citado 16 de octubre de 2016]. Recuperado a partir de: http://cmp.org.pe/wpcontent/uploads/2016/07/Norma_de_certificado_de_defuncion.pdf.

4. Pritt BS, Hardin NJ, Richmond JA, Shapiro SL. Death Certification Errors at an Academic Institution. Arch Pathol Lab Med. 2005;129:1476-9.

5. Inamadar PI, Vijayakumar BJ, Inamadar PP, Shobha. Evaluation of Different Errors in Writing Cause of Death Certificate. Indian Journal of Forensic Medicine \& Pathology. 2012; 5(1):17.

6. Rodriguez H, Ciriacos C, Piñeyrúa M, Logaldo R,
González D. Calidad del registro en el certificado de defunción en un hospital público de referencia. Montevideo, Uruguay, octubre-noviembre 2009. Rev Med Urug. 2010;26(4):216-23.

7. Cordero J, Nagai V, Santiago J, Costa R, Folha F, Nascimento F. Commitment in the completion of the medical death certification. Evaluation in Belém, Pará, Brazil in 2010. Rev Assoc Med Bras. 2013;59(4):335-40.

8. Soto-Cáceres V. Calidad del llenado de los certificados de defunción en un hospital público de Chiclayo, Perú 2006. Rev Peru Med Exp Salud Publica. 2008;25(3):330-32.

9. Valdez W, Gutiérrez C, Siura G. Análisis de la calidad de la certificación de defunciones en la región Ica, 2007. Rev. Peru. Epidemiol. 2013;17(1).

10. Rodríguez Martín O, Matos Valdivia Y, Anchia Alonso D, Betancourt Valladares M. Principales dificultades en el llenado de los certificados de defunción. Revista Cubana de Salud Pública. 2012;38(3):414-21.

11. Ministerio de Salud. Manual para el Ilenado de Formulario de Defunción. 2009 [consulta el 16 de marzo de 2016]; Recuperado a partir de: http:// www.limaeste.gob.pe/Virtual/estadistica/estadisticaData/DOCUMENTOS_NORMATIVOS/MANUAL_LLENADO_CERTIFICADO_DEFUNCIN.doc. 